Asia Pacific Journal of Mathematics, Vol. 5, No. 2 (2018), 233-242

\title{
I-CONVERGENT TRIPLE SEQUENCE SPACES OVER $n$-NORMED SPACE
}

\author{
TANWEER JALAL, ISHFAQ AHMAD MALIK* \\ Department of Mathematics, National Institute of Technology, Srinagar \\ Corresponding author: ishfaq_2phd15@nitsri.net
}

Received June 13, 2018

\begin{abstract}
The concept of 2-normed spaces was initially developed by Gähler [10], which was extend to $n$-norm by Misiak [17] for single sequence space. The main objective of this paper is to study triple sequence spaces over $n$-norm via the sequence of modulus functions. 2010 Mathematics Subject Classification. 40A05, 40C05, 46A45.
\end{abstract}

Key words and phrases. triple sequence spaces; I-convergence; modulus functions; ideal; $n$-normed space.

\section{INTRODUCTION}

A triple sequence (real or complex) is a function $x: \mathbb{N} \times \mathbb{N} \times \mathbb{N} \rightarrow \mathbb{R}(\mathbb{C})$, where $\mathbb{N}, \mathbb{R}$ and $\mathbb{C}$ are the set of natural numbers, real numbers, and complex numbers respectively. We denote by $\omega^{\prime \prime \prime}$ the class of all complex triple sequence $\left(x_{p q r}\right)$, where $p, q, r \in \mathbb{N}$. Then under the coordinate wise addition and scalar multiplication $\omega^{\prime \prime \prime}$ is a linear space. A triple sequence can be represented by a matrix, in case of double sequences we write in the form of a square. In case of triple sequence it will be in the form of a box in three dimensions.

The different types of notions of triple sequences and their statistical convergence were introduced and investigated initially by Sahiner et. al [22]. Later Debnath et.al [2, 3], Esi et.al $[4,5,6]$, Tripathy [24] and many others authors have studied it further and obtained various results.

Statistical convergence was introduced by Fast [7] and later on it was studied by Fridy $[8,9]$ from the sequence space point of view and linked it with summability theory. The notion of statistical convergent double sequence was introduced by Mursaleen and Edely [18].

(c)2018 Asia Pacific Journal of Mathematics 
I-convergence is a generalization of the statistical convergence. Kostyrko et. al. [15] introduced the notion of $I$-convergence of real sequence and studied its several properties. Later Jalal [11, 12, 13], Salat et. al. [20] and many other researchers contributed in its study. Sahiner and Tripathy [22] studied $I$-related properties in triple sequence spaces and showed some interesting results. Tripathy [24] extended the concept of $I$-convergent to double sequence and later Kumar [16] obtained some results on $I$-convergent double sequence. Recently Jalal and Malik [14] extended the concept of $n$-norms to triple sequence spaces and proved several algebraic and topological properties.

In this paper we define the spaces $c^{3}[\digamma,\|\cdot, \ldots,\|]^{I}, c_{0}^{3}[\digamma,\|\cdot, \ldots, \cdot\|]^{I}, \ell_{\infty}^{3}[\digamma,\|\cdot, \ldots, \cdot\|]^{I}$, $M_{I}^{3}[\digamma,\|\cdot, \ldots, \cdot\|]^{I}$ and $M_{0 I}^{3}[\digamma,\|\cdot, \ldots, \cdot\|]^{I}$ by using the concept of $n$-normed space via the sequence of modulii functions $F=\left(f_{p q r}\right)$. We study some algebraic and topological properties of these sequence spaces and some inclusion relations are obtained.

\section{DeFinitions AND PRELIMINARIES}

Definition 2.1. Let $X \neq \phi . A$ class $I \subset 2^{X}$ (Power set of $X$ ) is said to be an ideal in $X$ if the following conditions holds good:

(i) $I$ is additive that is if $A, B \in I$ then $A \cup B \in I$;

(ii) $I$ is hereditary that is if $A \in I$, and $B \subset A$ then $B \in I$.

$I$ is called non-trivial ideal if $X \notin I$

Definition 2.2. [21, 22] A triple sequence $\left(x_{p q r}\right)$ is said to be convergent to L in Pringsheim's sense if for every $\epsilon>0$, there exists $\mathbf{N} \in \mathbb{N}$ such that

$$
\left|x_{p q r}-L\right|<\epsilon \text { whenever } p \geq \mathbf{N}, q \geq \mathbf{N}, r \geq \mathbf{N}
$$

and write as $\lim _{p, p, r \rightarrow \infty} x_{p q r}=L$.

Note: A triple sequence is convergent in Pringsheim's sense may not be bounded [21, 22].

Example Consider the sequence $\left(x_{p q r}\right)$ defined by

$$
x_{p q r}=\left\{\begin{array}{cc}
p+q & \text { for all } p=q \text { and } r=1 \\
\frac{1}{p^{2} q r} & \text { otherwise }
\end{array}\right.
$$

Then $x_{p q r} \rightarrow 0$ in Pringsheim's sense but is unbounded.

Definition 2.3. A triple sequence $\left(x_{p q r}\right)$ is said to be I-convergence to a number $L$ if for every $\epsilon>0$,

$$
\left\{(p, q, r) \in \mathbb{N} \times \mathbb{N} \times \mathbb{N}:\left|x_{p q r}-L\right| \geq \epsilon\right\} \in I .
$$

In this case we write $I-\lim x_{p q r}=L$. 
Definition 2.4. A triple sequence $\left(x_{p q r}\right)$ is said to be $I$-null if $L=0$. In this case we write $I-\lim x_{p q r}=0$.

Definition 2.5. [21, 22] A triple sequence $\left(x_{p q r}\right)$ is said to be Cauchy sequence if for every $\epsilon>0$, there exists $\mathbf{N} \in \mathbb{N}$ such that

$$
\left|x_{p q r}-x_{l m n}\right|<\epsilon \text { whenever } p \geq l \geq \mathbf{N}, q \geq m \geq \mathbf{N}, r \geq n \geq \mathbf{N}
$$

Definition 2.6. A triple sequence $\left(x_{p q r}\right)$ is said to be $I-C a u c h y$ sequence if for every $\epsilon>0$, there exists $\mathbf{N} \in \mathbb{N}$ such that

$$
\left\{(p, q, r) \in \mathbb{N} \times \mathbb{N} \times \mathbb{N}:\left|x_{p q r}-a_{l m n}\right| \geq \epsilon\right\} \in I
$$

whenever $p \geq l \geq \mathbf{N}, q \geq m \geq \mathbf{N}, r \geq n \geq \mathbf{N}$

Definition 2.7. [21, 22] A triple sequence $\left(x_{p q r}\right)$ is said to be bounded if there exists $M>0$, such that $\left|x_{p q r}\right|<M$ for all $p, q, r \in \mathbb{N}$.

Definition 2.8. A triple sequence $\left(x_{p q r}\right)$ is said to be $I$-bounded if there exists $M>0$, such that $\left\{(p, q, r) \in \mathbb{N} \times \mathbb{N} \times \mathbb{N}:\left|x_{p q r}\right| \geq M\right\} \in I$ for all $p, q, r \in \mathbb{N}$.

Definition 2.9. A triple sequence space $E$ is said to be solid if $\left(\alpha_{p q r} x_{p q r}\right) \in E$ whenever $\left(x_{p q r}\right) \in E$ and for all sequences $\left(\alpha_{p q r}\right)$ of scalars with $\left|\alpha_{p q r}\right| \leq 1$, for all $p, q, r \in \mathbb{N}$.

Definition 2.10. Let $E$ be a triple sequence space and $x=\left(x_{p q r}\right) \in E$. Define the set $S(x)$ as

$$
S(x)=\left\{\left(x_{\pi(p q r)}\right): \pi \text { is a permutations of } \mathbb{N}\right\}
$$

If $S(x) \subseteq E$ for all $x \in E$, then $E$ is said to be symmetric.

Definition 2.11. A triple sequence space $E$ is said to be convergence free if $\left(y_{p q r}\right) \in E$ whenever $\left(x_{p q r}\right) \in E$ and $x_{p q r}=0$ implies $y_{p q r}=0$ for all $p, q, r \in \mathbb{N}$.

Definition 2.12. A triple sequence space $E$ is said to be sequence algebra if $x \cdot y \in E$, whenever $x=\left(x_{p q r}\right) \in E$ and $y=\left(y_{p q r}\right) \in E$, that is product of any two sequences is also in the space.

Definition 2.13. (n-Normed Space) Let $n \in \mathbb{N}$ and $X$ be a linear space over the field $\mathbb{R}$ of reals of dimension $d$, where $2 \leq d \leq n$. A real valued function $\|\cdot, \ldots, \cdot\|$ on $X^{n}$ satisfying the following four conditions:

(1) $\left\|x_{1}, x_{2}, \ldots, x_{n}\right\|=0$ if and only if $x_{1}, x_{2}, \ldots, x_{n}$ are linearly dependent in $X$;

(2) $\left\|x_{1}, x_{2}, \ldots, x_{n}\right\|$ is invariant under permutation;

(3) $\left\|\alpha x_{1}, x_{2}, \ldots, x_{n}\right\|=|\alpha|\left\|x_{1}, x_{2}, \ldots, x_{n}\right\|$ for any $\alpha \in \mathbb{R}$; 
(4) $\left\|x_{1}+x_{1}^{\prime}, x_{2}, \ldots, x_{n}\right\| \leq\left\|x_{1}, x_{2}, \ldots, x_{n}\right\|+\left\|x_{1}^{\prime}, x_{2}, \ldots, x_{n}\right\| ;$

is called an $n$-norm on $X$ and $(X,\|\cdot, \ldots, \cdot\|)$ is called an $n$-normed space over the field $\mathbb{R}$. For example $\left(\mathbb{R}^{n},\|\cdot, \ldots, \cdot\|_{E}\right)$ where $\left\|x_{1}, x_{2}, \ldots, x_{n}\right\|_{E}=$ the volume of the $n$-dimensional parallelopiped spanned by the vectors $x_{1}, x_{2}, \ldots, x_{n}$ Which can also be written as

$$
\left\|x_{1}, x_{2}, \ldots, x_{n}\right\|_{E}=\left|\operatorname{det}\left(x_{i j}\right)\right|
$$

where $x_{i}=\left(x_{i 1}, x_{i 2}, \cdots, x_{i n}\right) \in \mathbb{R}^{n}$ for each $i=1,2, \cdots, n$. Let $(X,\|\cdot, \ldots, \cdot\|)$ be an $n-$ normed space of dimension $2 \leq n \leq d$ and $\left\{a_{1}, a_{2}, \cdots, a_{n}\right\}$ be linearly independent set in $X$. Then the following function $\|\cdot, \ldots, \cdot\|_{\infty}$ on $X^{n-1}$ defined by

$$
\left\|x_{1}, x_{2}, \ldots, x_{n-1}\right\|_{\infty}=\max \left\{\left\|x_{1}, x_{2}, \ldots, x_{n-1}, a_{i}\right\|: i=1,2, \ldots, n\right\}
$$

defines an $(n-1)$-norm on $X$ with respect to $\left\{a_{1}, a_{2}, \ldots, a_{n}\right\}$.

The standard n-norm on $X$, a real inner product space of dimension $d \leq n$ is as follows:

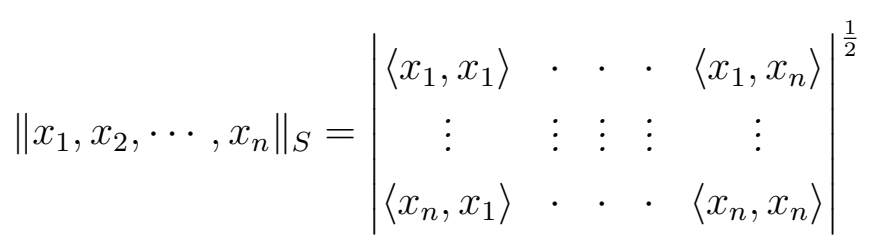

where $\langle\cdot, \cdot\rangle$ denotes the inner product on $X$. For $n=1$ this $n$-norm is the usual norm $\|x\|=\left\langle x_{1}, x_{1}\right\rangle^{\frac{1}{2}}$.

A sequence $\left(x_{k}\right)$ in a $n$-normed space $(X,\|\cdot, \ldots, \cdot\|)$ is said to converge to some $L \in X$ if

$$
\lim _{k \rightarrow \infty}\left\|x_{k}-L, z_{1}, \ldots, z_{n-1}\right\|=0 \text { for every } z_{1}, \ldots, z_{n-1} \in X .
$$

A sequence $\left(x_{k}\right)$ in a $n$-normed space $(X,\|\cdot, \ldots, \cdot\|)$ is said to be Cauchy if

$$
\lim _{k, p \rightarrow \infty}\left\|x_{k}-x_{p}, z_{1}, \ldots, z_{n-1}\right\|=0 \text { for every } z_{1}, \ldots, z_{n-1} \in X .
$$

If every Cauchy sequence in $X$ converges to some $L \in X$, then $X$ is said to be complete with respect to the $n$-norm. Any complete $n$-complete $n$-normed space is said to be $n$-Banach space. The n-normed space has been studied in stretch [6, 19, 23].

Definition 2.14. (Modulus Function) A function $f:[0, \infty) \rightarrow[0, \infty)$ is called a modulus function if it satisfies the following conditions

(i) $f(x)=0$ if and only if $x=0$.

(ii) $f(x+y) \leq f(x)+f(y)$ for all $x \geq 0$ and $y \geq 0$.

(iii) $f$ is increasing.

(iv) $f$ is continuous from the right at 0 . 
Since $|f(x)-f(y)| \leq f(|x-y|)$, it follows from condition (iv) that $f$ is continuous on $[0, \infty)$. Furthermore, from condition (2) we have $f(n x) \leq n f(x)$, for all $n \in \mathbb{N}$, and so $f(x)=f\left(n x\left(\frac{1}{n}\right)\right) \leq n f\left(\frac{x}{n}\right)$.

Hence $\frac{1}{n} f(x) \leq f\left(\frac{x}{n}\right)$ for all $n \in \mathbb{N}$

Let $I$ be an admissible ideal, $F=\left(f_{p q r}\right)$ be a sequence of modulus functions and $(X,\|\cdot, \ldots, \cdot\|)$ be a $n$-normed space. By $\omega^{\prime \prime \prime}(n-X)$ we denote the space of all triple sequences defined over $(X,\|\cdot, \ldots, \cdot\|)$. In the present paper we define the following sequence spaces

$$
\begin{gathered}
c^{3}[F,\|\cdot, \ldots,\| \|]^{I}=\left\{x=x_{p q r} \in \omega^{\prime \prime \prime}(n-X): \forall \epsilon>0, \text { the set }\{(p, q, r) \in \mathbb{N} \times \mathbb{N} \times \mathbb{N}:\right. \\
\left.\left.f_{p q r}\left(\left\|x_{p q r}-L, z_{1}, \cdots, z_{n-1}\right\|\right) \geq \epsilon, \text { for some } L \in \mathbb{C} \text { and } z_{1}, \ldots, z_{n-1} \in X\right\} \in I\right\} \\
c_{0}^{3}[F,\|\cdot, \ldots, \cdot\|]^{I}=\left\{x=x_{p q r} \in \omega^{\prime \prime \prime}(n-X): \forall \epsilon>0, \text { the set }\{(p, q, r) \in \mathbb{N} \times \mathbb{N} \times \mathbb{N}:\right. \\
\left.\left.f_{p q r}\left(\left\|x_{p q r}, z_{1}, \cdots, z_{n-1}\right\|\right) \geq \epsilon, z_{1}, \ldots, z_{n-1} \in X\right\} \in I\right\} \\
\ell_{\infty}^{3}[F,\|\cdot, \ldots, \cdot\|]^{I}=\left\{x=x_{p q r} \in \omega^{\prime \prime \prime}(n-X): \exists K>0 \text { such that }\{(p, q, r) \in \mathbb{N} \times \mathbb{N} \times \mathbb{N}:\right. \\
\left.\left.\sup _{p, q, r \geq 1}\left\{f_{p q r}\left(\left\|x_{p q r}, z_{1}, \cdots, z_{n-1}\right\|\right)\right\} \geq K, z_{1}, \ldots, z_{n-1} \in X\right\} \in I\right\}
\end{gathered}
$$

and

$$
\begin{aligned}
& M^{3}[F,\|\cdot, \ldots, \cdot\|]^{I}=c^{3}[F,\|\cdot, \ldots, \cdot\|]^{I} \cap \ell_{\infty}^{3}[F,\|\cdot, \ldots, \cdot\|]^{I} \\
& M_{0}^{3}[F,\|\cdot, \ldots, \cdot\|]^{I}=c_{0}^{3}[F,\|\cdot, \ldots, \cdot\|]^{I} \cap \ell_{\infty}^{3}[F,\|\cdot, \ldots, \cdot\|]^{I}
\end{aligned}
$$

For $F(x)=x$ we have

$$
\begin{gathered}
c^{3}[\|\cdot, \ldots, \cdot\|]^{I}=\left\{x=x_{p q r} \in \omega^{\prime \prime \prime}(n-X): \forall \epsilon>0, \text { the set }\{(p, q, r) \in \mathbb{N} \times \mathbb{N} \times \mathbb{N}:\right. \\
\left.\left.\left\|x_{p q r}-L, z_{1}, \cdots, z_{n-1}\right\| \geq \epsilon, \text { for some } L \in \mathbb{C} \text { and } z_{1}, \ldots, z_{n-1} \in X\right\} \in I\right\} \\
c_{0}^{3}[\|\cdot, \ldots, \cdot\|]^{I}=\left\{x=x_{p q r} \in \omega^{\prime \prime \prime}(n-X): \forall \epsilon>0, \text { the } \operatorname{set}\{(p, q, r) \in \mathbb{N} \times \mathbb{N} \times \mathbb{N}:\right. \\
\left.\left.\left\|x_{p q r}, z_{1}, \cdots, z_{n-1}\right\| \geq \epsilon, z_{1}, \ldots, z_{n-1} \in X\right\} \in I\right\} \\
\ell_{\infty I}^{3}[\|\cdot, \ldots, \cdot\|]^{I}=\left\{x=x_{p q r} \in \omega^{\prime \prime \prime}(n-X): \exists K>0 \text { such that }\{(p, q, r) \in \mathbb{N} \times \mathbb{N} \times \mathbb{N}:\right. \\
\left.\left.\sup _{p, q, r \geq 1}\left(\left\|x_{p q r}, z_{1}, \cdots, z_{n-1}\right\|\right) \geq K, z_{1}, \ldots, z_{n-1} \in X\right\} \in I\right\}
\end{gathered}
$$


and

$$
\begin{aligned}
& M^{3}[\|\cdot, \ldots, \cdot\|]^{I}=c^{3}[\|\cdot, \ldots, \cdot\|]^{I} \cap \ell_{\infty}^{3}[\|\cdot, \ldots, \cdot\|]^{I} \\
& M_{0}^{3}[\|\cdot, \ldots, \cdot\|]^{I}=c_{0}^{3}[\|\cdot, \ldots, \cdot\|]^{I} \cap \ell_{\infty}^{3}[\|\cdot, \ldots, \cdot\|]^{I}
\end{aligned}
$$

\section{Algebraic and Topological Properties of the new Sequence spaces}

Theorem 3.1. Let $F=\left(f_{p q r}\right)$ be a sequence of modulus functions then the triple sequence $\operatorname{spaces}_{0}^{3}[F,\|\cdot, \ldots, \cdot\|]^{I}, c^{3}[F,\|\cdot, \ldots, \cdot\|]^{I}, \ell_{\infty}^{3}[F,\|\cdot, \ldots, \cdot\|]^{I}, M^{3}[F,\|\cdot, \ldots, \cdot\|]^{I}$ and $M_{0}^{3}[F,\|\cdot, \ldots, \cdot\|]^{I}$ all linear over the field $\mathbb{C}$ of complex numbers.

Proof. We prove the result for the sequence space $c^{3}[\digamma,\|\cdot, \ldots, \cdot\|]^{I}$.

Let $x=\left(x_{p q r}\right), y=\left(y_{p q r}\right) \in c^{3}[\digamma,\|\cdot, \ldots, \cdot\|]^{I}$ and $\alpha, \beta \in \mathbb{C}$, then there exist positive integers $m_{\alpha}$ and $n_{\beta}$ such that $|\alpha| \leq m_{\alpha}$ and $|\beta| \leq n_{\beta}$, then for $z_{1}, z_{2}, \ldots, z_{n-1} \in X$

$$
\begin{aligned}
& I-\lim f_{p q r}\left(\left\|x_{p q r}-L_{1}, z_{1}, \ldots, z_{n-1}\right\|\right)=0, \text { for some } L_{1} \in \mathbb{C} \\
& I-\lim f_{p q r}\left(\left\|x_{p q r}-L_{2}, z_{1}, \ldots, z_{n-1}\right\|\right)=0, \text { for some } L_{2} \in \mathbb{C}
\end{aligned}
$$

Now for a given $\epsilon>0$ we set

$$
\begin{aligned}
& C_{1}=\left\{(p, q, r) \in \mathbb{N} \times \mathbb{N} \times \mathbb{N}: f_{p q r}\left(\left\|x_{p q r}-L_{1}, z_{1}, \ldots, z_{n-1}\right\|\right)>\frac{\epsilon}{2}\right\} \in I \\
& C_{2}=\left\{(p, q, r) \in \mathbb{N} \times \mathbb{N} \times \mathbb{N}: f_{p q r}\left(\left\|y_{p q r}-L_{2}, z_{1}, \ldots, z_{n-1}\right\|\right)>\frac{\epsilon}{2}\right\} \in I
\end{aligned}
$$

Since $f_{p q r}$ is a modulus function, so it is non-decreasing and convex, hence we get

$$
\begin{aligned}
f_{p q r}\left(\|\left(\alpha x_{p q r}+\beta y_{p q r}\right)-\right. & \left.\left(\alpha L_{1}+\beta L_{2}\right), z_{1}, \ldots, z_{n-1} \|\right) \\
& =f_{p q r}\left(\left\|\left(\alpha x_{p q r}-\alpha L_{1}\right)+\left(\beta y_{p q r}-\beta L_{2}\right), z_{1}, \ldots, z_{n-1}\right\|\right) \\
& \leq f_{p q r}\left(|\alpha|\left\|x_{p q r}-L_{1}, z_{1}, \ldots, z_{n-1}\right\|\right)+f_{p q r}\left(|\beta|\left\|y_{p q r}-L_{2}, z_{1}, \ldots, z_{n-1}\right\|\right) \\
& =|\alpha| f_{p q r}\left(\left|x_{p q r}-L_{1}\right|\right)+|\beta| f_{p q r}\left(\left|y_{p q r}-L_{2}\right|\right) \\
& \leq m_{\alpha} f_{p q r}\left(\left\|x_{p q r}-L_{1}, z_{1}, \ldots, z_{n-1}\right\|\right)+n_{\beta} f_{p q r}\left(\left\|y_{p q r}-L_{2}, z_{1}, \ldots, z_{n-1}\right\|\right)
\end{aligned}
$$

From (2.1) and (2.2) we can write

$$
\left\{(p, q, r) \in \mathbb{N} \times \mathbb{N} \times \mathbb{N}: f_{p q r}\left(\left\|\left(\alpha x_{p q r}+\beta y_{p q r}\right)-\left(\alpha L_{1}+\beta L_{2}\right), z_{1}, \ldots, z_{n-1}\right\|\right)>\epsilon\right\} \subseteq C_{1} \cup C_{2}
$$

Thus $\alpha x+\beta y \in c^{3}[\digamma,\|\cdot, \ldots, \cdot\|]^{I}$.

Therefore $c^{3}[\digamma,\|\cdot, \ldots, \cdot\|]^{I}$ is a linear space.

In the same way we can show that other spaces are linear as well. 
Theorem 3.2. Let $\digamma=\left(f_{\text {pqr }}\right)$ be a sequence of modulus functions then the inclusions $c_{0}^{3}[\digamma,\|\cdot, \ldots, \cdot\|]^{I} \subset c^{3}[\digamma,\|\cdot, \ldots, \cdot\|]^{I} \subset \ell_{\infty}^{3}[\digamma,\|\cdot, \ldots, \cdot\|]^{I}$ holds.

Proof. The inclusion $c_{0}^{3}[\digamma,\|\cdot, \ldots, \cdot\|]^{I} \subset c^{3}[\digamma,\|\cdot, \ldots, \cdot\|]^{I}$ is obvious.

We prove $c^{3}[\digamma,\|\cdot, \ldots, \cdot\|]^{I} \subset \ell_{\infty}^{3}[\digamma,\|\cdot, \ldots, \cdot\|]^{I}$.

Let $x=\left(x_{p q r}\right) \in c^{3}[\digamma,\|\cdot, \ldots, \cdot\|]^{I}$ then there exists $L \in \mathbb{C}$ such that $I-\lim f_{p q r}\left(\left\|x_{p q r}-L, z_{1}, \ldots, z_{n-1}\right\|\right)=0, z_{1}, \ldots, z_{n-1} \in X$

Since $\digamma=\left(f_{p q r}\right)$ is a sequence of modulus functions so

$$
f_{p q r}\left(\left\|x_{p q r}, z_{1}, \ldots, z_{n-1}\right\|\right) \leq f_{p q r}\left(\left\|x_{p q r}-L, z_{1}, \ldots, z_{n-1}\right\|\right)+f_{p q r}\left(\left\|L, z_{1}, \ldots, z_{n-1}\right\|\right)
$$

On taking supremum over $p, q$ and $r$ on both sides gives $x=\left(x_{p q r}\right) \in \ell_{\infty}^{3}[\digamma,\|\cdot, \ldots, \cdot\|]^{I}$ Hence the inclusion $c_{0}^{3}[\digamma,\|\cdot, \ldots, \cdot\|]^{I} \subset c^{3}[\digamma,\|\cdot, \ldots, \cdot\|]^{I}$ $\subset \ell_{\infty}^{3}[\digamma,\|\cdot, \ldots, \cdot\|]^{I}$ holds.

Theorem 3.3. The triple sequence $c_{0}^{3}[\digamma,\|\cdot, \ldots, \cdot\|]^{I}$ and $M_{0}^{3}[\digamma,\|\cdot, \ldots, \cdot\|]^{I}$ are solid.

Proof. We prove the result for $c_{0}^{3}[\digamma,\|\cdot, \ldots, \cdot\|]^{I}$.

Consider $x=\left(x_{p q r}\right) \in c_{0}^{3}[\digamma,\|\cdot, \ldots, \cdot\|]^{I}$, then $I-\lim _{p, q, r} f_{p q r}\left(\left\|x_{p q r}, z_{1}, \ldots, z_{n-1}\right\|\right)=0$

Consider a sequence of scalar $\left(\alpha_{p q r}\right)$ such that $\left|\alpha_{p q r}\right| \leq 1$ for all $p, q, r \in \mathbb{N}$.

Then we have

$$
\begin{aligned}
I-\lim _{p, q, r} f_{p q r}\left(\mid \alpha_{p q r}\left(x_{p q r}\right), z_{1}, \ldots, z_{n-1} \|\right) & \leq I-\left|\alpha_{p q r}\right| \lim _{p, q, r} f_{p q r}\left(\left\|x_{p q r}, z_{1}, \ldots, z_{n-1}\right\|\right) \\
& \leq I-\lim _{p, q, r} f_{p q r}\left(\left\|x_{p q r}, z_{1}, \ldots, z_{n-1}\right\|\right) \\
& =0
\end{aligned}
$$

Hence $I-\lim _{p, q, r} f_{p q r}\left(\left\|\alpha_{p q r} x_{p q r}, z_{1}, \ldots, z_{n-1}\right\|\right)=0$ for all $p, q, r \in \mathbb{N}$

Which gives $\left(\alpha_{p q r} x_{p q r}\right) \in c_{0}^{3}[\digamma,\|\cdot, \ldots, \cdot\|]^{I}$

Hence the sequence space $c_{0}^{3}[\digamma,\|\cdot, \ldots, \cdot\|]^{I}$ is solid.

The result for $M_{0}^{3}[\digamma,\|\cdot, \ldots, \cdot\|]^{I}$ can be similarly proved.

Theorem 3.4. The triple sequence spaces $c_{0}^{3}[\digamma,\|\cdot, \ldots, \cdot\|]^{I}, c^{3}[\digamma,\|\cdot, \ldots, \cdot\|]^{I}, \ell_{\infty}^{3}[\digamma,\|\cdot, \ldots, \cdot\|]^{I}$ ,$M^{3}[\digamma,\|\cdot, \ldots, \cdot\|]^{I}$ and $M_{0}^{3}[\digamma,\|\cdot, \ldots, \cdot\|]^{I}$ are sequence algebras.

Proof. We prove the result for $c_{0}^{3}[\digamma,\|\cdot, \ldots, \cdot\|]^{I}$.

Let $x=\left(x_{p q r}\right), y=\left(y_{p q r}\right) \in c_{0}^{3}[\digamma,\|\cdot, \ldots, \cdot\|]^{I}$

Then we have $I-\lim f_{p q r}\left(\left\|x_{p q r}, z_{1}, \ldots, z_{n-1}\right\|\right)=0$ and $I-\lim f_{p q r}\left(\left\|x_{p q r}, z_{1}, \ldots, z_{n-1}\right\|\right)=0$ Using definition modulus functions we have $I-\lim f_{p q r}\left(\left\|\left(x_{p q r} \cdot y_{p q r}\right), z_{1}, \ldots, z_{n-1}\right\|\right)=0$. It 
implies that $x \cdot y \in c_{0}^{3}[\digamma,\|\cdot, \ldots, \cdot\|]^{I}$

The result can be proved for the spaces $c^{3}[\digamma,\|\cdot, \ldots,\|]^{I}, \ell_{\infty}^{3}[\digamma,\|\cdot, \ldots, \cdot\|]^{I}, M^{3}[\digamma,\|\cdot, \ldots, \cdot\|]^{I}$ and $M_{0}^{3}[\digamma,\|\cdot, \ldots, \cdot\|]^{I}$ in the same way.

Theorem 3.5. In general the sequence $\operatorname{spacesc}_{0}^{3}[\digamma,\|\cdot, \ldots, \cdot\|]^{I}, c^{3}[\digamma,\|\cdot, \ldots, \cdot\|]^{I}$ and $\ell_{\infty}^{3}[\digamma,\|\cdot, \ldots, \cdot\|]^{I}$ are not convergence free.

Proof. We prove the result for the sequence space $c_{I}^{3}[\digamma,\|\cdot, \ldots, \cdot\|]^{I}$ using an example.

Example: Let $I=I_{f}$ define the triple sequence $x=\left(x_{p q r}\right)$ as

$$
x_{p q r}=\left\{\begin{array}{lc}
0 & \text { if } p=q=r \\
1 & \text { otherwise }
\end{array}\right.
$$

Then if $f_{p q r}(x)=x_{p q r} \forall p, q, r \in \mathbb{N}$, we have $x=\left(x_{p q r}\right) \in c^{3}[\digamma,\|\cdot, \ldots, \cdot\|]^{I}$.

Now define the sequence $y=y_{p q r}$ as

$$
y_{p q r}=\left\{\begin{array}{cc}
0 & \text { if } r \text { is odd }, \text { and } p, q \in \mathbb{N} \\
\operatorname{lm} n & \text { otherwise }
\end{array}\right.
$$

Then for $f_{p q r}(x)=x_{p q r} \forall p, q, r \in \mathbb{N}$, it is clear that $y=\left(y_{p q r}\right) \notin c^{3}[\digamma,\|\cdot, \ldots, \cdot\|]^{I}$

Hence the sequence spaces $c^{3}[\digamma,\|\cdot, \ldots, \cdot\|]^{I}$ is not convergence free.

The space $c^{3}[\digamma,\|\cdot, \ldots, \cdot\|]^{I}$ and $\ell_{\infty}^{3}[\digamma,\|\cdot, \ldots, \cdot\|]^{I}$ are not convergence free in general can be proved in the same fashion.

Theorem 3.6. In general the triple sequences $c_{0}^{3}[\digamma,\|\cdot, \ldots, \cdot\|]^{I}$ and $c^{3}[\digamma,\|\cdot, \ldots, \cdot\|]^{I}$ are not symmetric if $I$ is neither maximal nor $I=I_{f}$.

Proof. We prove the result for the sequence space $c_{0}^{3}[\digamma,\|\cdot, \ldots, \cdot\|]^{I}$ using an example.

Example: Define the triple sequence $x=\left(x_{p q r}\right)$ as

$$
x_{p q r}=\left\{\begin{array}{cc}
0 & \text { if } r=1, \text { for all } p, q \in \mathbb{N} \\
1 & \text { otherwise }
\end{array}\right.
$$

Then if $f_{p q r}(x)=x_{p q r} \forall p, q, r \in \mathbb{N}$, we have $x=\left(x_{p q r}\right) \in c_{0}^{3}[\digamma,\|\cdot, \ldots, \cdot\|]^{I}$.

Now if $x_{\pi(p q r)}$ be a rearrangement of $x=\left(x_{p q r}\right)$ defined as

$$
x_{\pi(p q r)}=\left\{\begin{array}{lc}
1 & \text { for } \\
0 & \text { otherwise }
\end{array}\right.
$$

Then $\left\{x_{\pi(p, q, r)}\right\} \notin c_{0}^{3}[\digamma,\|\cdot, \ldots, \cdot\|]^{I}$ as $x_{\pi(p q r)}=1$

Hence the sequence spaces $c_{0}^{3}[\digamma,\|\cdot, \ldots, \cdot\|]^{I}$ is not symmetric in general.

The space $c^{3}[\digamma,\|\cdot, \ldots, \cdot\|]^{I}$ is not symmetric in general can be proved in the same fashion. 
Theorem 3.7. Let $F=\left(f_{p q r}\right)$ and $G=\left(g_{p q r}\right)$ be two sequences of modulus functions. Then

$$
\mathcal{T}^{3}[F,\|\cdot, \ldots, \cdot\|]^{I} \cap \mathcal{T}^{3}[G,\|\cdot, \ldots, \cdot\|]^{I} \subseteq \mathcal{T}^{3}[F+G,\|\cdot, \ldots, \cdot\|]^{I}
$$

where $\mathcal{T}=c, c_{0}$, or $\ell_{\infty}$

Proof. We prove the result for $\mathcal{T}=\ell_{\infty}$. Let $x=\left(x_{i j k}\right) \in \ell_{\infty}^{3}[F,\|\cdot, \ldots, \cdot\|]^{I} \cap \ell_{\infty}^{3}[G,\|\cdot, \ldots, \cdot\|]^{I}$. Then for $z_{1}, \ldots, z_{n-1} \in X$ we have

$$
\left\{(p, q, r) \in \mathbb{N} \times \mathbb{N} \times \mathbb{N}: \sup _{p, q, r \geq 1}\left\{f_{p q r}\left(\left\|x_{p q r}, z_{1}, \cdots, z_{n-1}\right\|\right)\right\} \geq K_{1}\right\} \in I \text { for some } K_{1}>0
$$

and

$$
\left\{(p, q, r) \in \mathbb{N} \times \mathbb{N} \times \mathbb{N}: \sup _{p, q, r \geq 1}\left\{g_{p q r}\left(\left\|x_{p q r}, z_{1}, \cdots, z_{n-1}\right\|\right)\right\} \geq K_{2}\right\} \in I \text { for some } K_{2}>0
$$

Now since

$$
\begin{array}{r}
\sup _{p, q, r \geq 1}\left\{\left(f_{p q r}+g_{p q r}\right)\left(\left\|x_{p q r}, z_{1}, \cdots, z_{n-1}\right\|\right)\right\}=\sup _{p, q, r \geq 1}\left\{f_{p q r}\left(\left\|x_{p q r}, z_{1}, \cdots, z_{n-1}\right\|\right)+g_{p q r}\left(\left\|x_{p q r}, z_{1}, \cdots, z_{n-1}\right\|\right)\right\} \\
\leq \sup _{p, q, r \geq 1}\left\{f_{p q r}\left(\left\|x_{p q r}, z_{1}, \cdots, z_{n-1}\right\|\right)\right\}+\sup _{p, q, r \geq 1}\left\{g_{p q r}\left(\left\|x_{p q r}, z_{1}, \cdots, z_{n-1}\right\|\right)\right\}
\end{array}
$$

Hence for $K=\max \left\{K_{1}, K_{2}\right\}$ we have

$$
\left\{(p, q, r) \in \mathbb{N} \times \mathbb{N} \times \mathbb{N}: \sup _{p, q, r \geq 1}\left\{\left(f_{p q r}+g_{p q r}\right)\left(\left\|x_{p q r}, z_{1}, \cdots, z_{n-1}\right\|\right)\right\} \geq K\right\} \in I
$$

Therefore $x \in \ell_{\infty}^{3}[F+G,\|\cdot, \ldots, \cdot\|]^{I}$.

Hence

$$
\ell_{\infty}^{3}[F,\|\cdot, \ldots, \cdot\|]^{I} \cap \ell_{\infty}^{3}[G,\|\cdot, \ldots, \cdot\|]^{I} \subseteq \ell_{\infty}^{3}[F+G,\|\cdot, \ldots, \cdot\|]^{I}
$$

In the same way the inclusion for $\mathcal{T}=c, c_{0}$ can be proved.

\section{REFERENCES}

[1] A. Alotaibi, M. Mursaleen and S. K. Sharma, Double sequence spaces over n-normed spaces defined by a sequence of orlicz functions, J. Inequal. Appl., 2014 (2014), Article ID 216.

[2] S. Debnath, B. Sarma, B.C. Das, Some generalized triple sequence spaces of real numbers J. Nonlinear Anal. Optim. 6 (2015), 71-79.

[3] S. Debnath and N. Subramanian, Generalized rough lacunary statistical triple difference sequence spaces in probability of fractional order defined by musielak-orlicz function Bol. Soc. Paran. Mat. (3s) Vol. 37, (2019), 5562.

[4] A. Esi, On some triple almost lacunary sequence spaces defined by orlicz functions, Res. Rev., Discr. Math. Struct. 1 (2014), 16-25.

[5] A. Esi and M.N. Catalbas, Almost convergence of triple sequences G. J. Math. Anal. 2, (2014), 6-10.

[6] A. Esi and E. Savas On lacunary statically convergent triple sequences in probabilistic normed space Appl Mathand Inf Sci 9, (2015), 2529-2534.

[7] H. Fast, Surla convergence statistique, Colloq. Math., 2, (1951), 241-244. 
[8] J.A. Fridy, On statistical convergence, Analysis, 5, (1985), 301-313.

[9] J.A. Fridy, Statistical limit points, Proc. Amer. Math. Soc., 11, (1993), 1187-1192.

[10] S. Gähler, Linear 2-normietre Rume, Math. Nachr., 28 (1965), 1-43.

[11] T. Jalal, Some new I-convergent sequence spaces defined by using a sequence of modulus functions in n-normed spaces, Int. J. Math. Archive, 5(9) (2014), 202-209.

[12] T. Jalal, Some new I-lacunary generalized difference sequence spaces defined in n-normed spaces, Springer Proc. Math. Sat.,171 (2016), 249-258.

[13] T. Jalal, Some new lacunary sequence spaces of invariant means defined by musielak-Orlicz functions on n-normed space, Int. J. P. Appl. Math., 119(7) (2018), 1-11.

[14] T. Jalal and I.A. Malik, Some new triple sequence spaces over n-normed space, Proyecciones, (2018), (to appear).

[15] P. Kostyrko, T. Salat, W. Wilczynski, I-convergence, Real Anal. Exch. 26(2) (2000), 669-686.

[16] V. Kumar, On I-convergence of double sequences, Math. Commun.,12 (2007), 171-181.

[17] A. Misiak, n-Inner product spaces, Math. Nachr., 140 (1989), 299-319.

[18] M. Mursaleen and O. H. H. Edely, Statistical convergence of double sequences, J. Math. Anal. Appl., 288 (2003), 223-231.

[19] M. Mursaleen, K. Raj and S. K. Sharma, Some spaces of differences and lacunary statistical convergence in n-normed space defined by sequence of orlicz functions, Miskolc Math. Notes, 16(1) (2015), 283-304.

[20] T. Salat, B. C. Tripathy and M. Ziman, On some properties of I-convergence, Tatra Mountain Math. Publ., (2000) 669-686.

[21] A. Sahiner, M. Gurdal and F.K. Duden, Triple Sequences and their statistical convergence, Selcuk J. Appl Math 8, (2007), 49-55.

[22] A. Sahiner and B.C. Tripathy, Some I related properties of triple sequences, Selcuk J. Appl. Math. 9 (2008), 9-18.

[23] S.K. Sharma and Ayhan Esi, Some I-convergent sequence spaces defined by using sequence of moduli and n-normed space, J. Egyp. Math. Soc. 21(2) (2013), 103-107.

[24] B. C. Tripathy, Statistically convergent double sequence, Tamkang. J. Math., 34(3) (2003), 231-237. 